\title{
NECESSARY AND SUFFICIENT CONDITIONS FOR GLOBAL-IN-TIME EXISTENCE OF SOLUTIONS OF ORDINARY, STOCHASTIC, AND PARABOLIC DIFFERENTIAL EQUATIONS
}

YURI E. GLIKLIKH

Received 26 June 2005; Accepted 1 July 2005

We derive necessary and sufficient conditions for global-in-time existence of solutions of ordinary differential, stochastic differential, and parabolic equations. The conditions are formulated in terms of complete Riemannian metrics on extended phase spaces (conditions with two-sided estimates) or in terms of derivatives of proper functions on extended phase spaces (conditions with one-sided estimates).

Copyright (C) 2006 Yuri E. Gliklikh. This is an open access article distributed under the Creative Commons Attribution License, which permits unrestricted use, distribution, and reproduction in any medium, provided the original work is properly cited.

\section{Introduction}

This is a survey paper with complete proofs of results obtained in $[6,7,9-11]$. We derive necessary and sufficient conditions for global-in-time existence of solutions of ordinary, stochastic, and parabolic differential equations. They are obtained as modifications of some well-known sufficient conditions (both with one-sided and two-sided estimates). In particular those modifications involve transition to extended phase spaces. We consider the general case of equations on smooth manifolds (mainly finite-dimensional). For ordinary differential equations we get necessary and sufficient conditions of both two-sided and one-sided sorts (in the latter case we also get a generalization to a certain infinite-dimensional case). For stochastic differential and parabolic equations we obtain necessary and sufficient conditions of one-sided sort for some classes of equations on finite-dimensional manifolds.

Recall that if all solutions of Cauchy problems of an ordinary differential equation with a smooth vector field in the right-hand side on a finite-dimensional manifold $M$ exist on the entire line $(-\infty, \infty)$, the vector field and its flow are called complete. Below the solutions to Cauchy problems will be called the orbits of the flow or the integral curves of the vector field.

If the manifold $M$ is compact, all continuous (in particular, all smooth) vector fields are complete. Indeed, in this case any Riemannian metric on $M$ is complete, any continuous vector field is bounded, hence any integral curve has bounded length on any finite 
interval, that is, it is relatively compact. Thus, the flow of a smooth vector field is a diffeomorphism of $M$ onto $M$ at any time instant belonging to $(-\infty,+\infty)$, that is, the flow is a flow of diffeomorphisms.

In the case of noncompact manifolds (in particular, in linear spaces) the integral curves can get to infinity within some finite time interval and the problem of the flow completeness becomes nontrivial. Analogous situation takes place also for stochastic differential and parabolic equations.

Plenty of sufficient conditions for completeness of the flows of ordinary differential equations in linear spaces are well known. There exist two sorts of such conditions: with two-sided estimates and with one-sided estimates. The former is formulated in terms of estimates on the norm of the right-hand side and guarantees the existence of all integral curves for $t \in(-\infty,+\infty)$. Let us present some examples.

Let $X(t, x)$ be a smooth vector field on $R^{n}$. Consider the differential equation

$$
\dot{x}(t)=X(t, x(t))
$$

The simplest examples of conditions with two-sided estimates are

(i) $\|X(t, x)\|<\psi(t)$ at all $x \in R^{n}$ and $t \in(-\infty, \infty)$ for some function $\psi>0$ that is integrable on any finite interval (boundedness);

(ii) $\|X(t, x)\|<\psi(t)(1+\|x\|)$ with analogous $\psi$ (linear growth).

The Wintner's theorem proves the completeness under the following conditions: $\|X(t, x)\|<\psi(t) L(\|x\|)$ where $\psi>0$ is as above and $L:[0, \infty) \rightarrow(0, \infty)$ is a continuous function such that

$$
\int_{0}^{\infty} \frac{1}{L(u)} d u=\infty
$$

On nonlinear smooth manifolds analogous conditions are formulated in terms of norms generated by complete Riemannian metrics. Notice that under the conditions of Wintner's theorem we can take a certain smooth approximation of $L$ (denote it also by $L)$, such that (1.2) is valid for it, and introduce the new Riemannian metric on $R^{n}$ by the formula $\langle\cdot, \cdot\rangle_{x}=\left(1 / L(\|x\|)^{2}\right)(\cdot, \cdot)$ where $\langle\cdot, \cdot\rangle_{x}$ is the Riemanninan scalar product in the tangent space $T_{x} R^{n}$ and $(\cdot, \cdot)$ is the Euclidean scalar product in $R^{n}$. From condition (1.2) one can easily derive that the new Riemannian metric is complete. Thus, the condition of Wintner's theorem means boundedness with respect to the new complete Riemannian metric in $R^{n}$. Notice also that the condition of linear growth is a particular case the Wintner's one and so for it there also exists a complete Riemannian metric with respect to which the right-hand side is uniformly bounded.

An example of conditions with one-sided estimates in $R^{n}$ is as follows. Let $\varphi: R^{n} \rightarrow R$ be a smooth positive function such that $\varphi(x) \rightarrow \infty$ as $x \rightarrow \infty$. Then all integral curves exist for $t \in\left(t_{0}, \infty\right)$ (where $t_{0}$ is the initial time value of the curve) if $(X(t, x), \operatorname{grad} \varphi)<C$ at all $t \in\left(t_{0}, \infty\right), x \in R^{n}$ for some real constant $C$. Notice that such conditions guarantee existence from any specified finite time instant to $+\infty$ but for $t \rightarrow-\infty$ the solutions may get to infinity within a finite time interval. Below we consider a modification of these conditions like $|(X(t, x), \operatorname{grad} \varphi)|<C$ for a certain $C>0$ that guarantees existence of all 
integral curves on $(-\infty,+\infty)$. Such conditions we will also call the ones with one-sided estimates.

To describe the conditions with one-sided estimates on manifolds, notice that the property $\varphi(x) \rightarrow \infty$ as $x \rightarrow \infty$ means that $\varphi$ is a so-called proper function on $R^{n}$, that is, its preimage of any relatively compact set in $R$ is relatively compact in $R^{n}$ (see general definition for proper functions on manifolds below). On the other hand, the product $(X(t, x), \operatorname{grad} \varphi)$ is equal to the derivative $X \varphi$ of $\varphi$ in the direction of vector field $X$ at $x \in R^{n}$. Thus, a condition with one-sided estimate on a smooth manifold $M$ can be formulated as follows: let there exist a smooth proper positive function $\varphi$ on $M$ such that $|X \varphi|<C$ for some positive constant $C$ at all $t \in(-\infty, \infty), m \in M$. Then all integral curves of $X$ exist on $(-\infty, \infty)$. Obviously for the condition $X \varphi<C$ we will get completeness in going only forward.

Analogous conditions with one-sided and two-sided estimates are known for completeness of flows of stochastic differential equations. An example of conditions with two-sided estimates is the well-known Itô condition of linear growth (see, e.g., [5]). In conditions of one-sided type for stochastic differential equations the operator of derivative in the direction of vector field in the right-hand side is replaced by the generator of stochastic flow, a special second-order differential operator. Among sufficient conditions of this sort we mention Elworthy's condition from [3, Theorem IX. 6A] and its particular case from Theorem 5.3 below. We discuss the stochastic case in more detail in Section 5 .

For parabolic equations analogous sufficient conditions are also known. In particular, they can be obtained in the framework of stochastic approach to parabolic equations.

As it is mentioned above, in this paper we find modifications of sufficient conditions of completeness that make them necessary and sufficient. The structure of the paper is as follows. In Section 2 we deal with necessary and sufficient conditions of two-sided sort for completeness of smooth vector fields on finite-dimensional manifolds. Section 3 is devoted to the same problem but for conditions of one-sided sort. In Section 4 we obtain a generalization of conditions from Section 3 to a certain infinite-dimensional case. In Section 5 we get a necessary and sufficient condition of one-sided sort for completeness of a stochastic flow, continuous at infinity, on a finite-dimensional manifold. In Section 6, from the results of Section 5 we derive necessary and sufficient conditions for existence of global Feller semigroup for parabolic equations of some special type on finite-dimensional manifolds.

Preliminary information can be seen, for example, in [8].

\section{Necessary and sufficient conditions of two-sided type for completeness of ODE flows}

As we have mentioned in Section 1, under the conditions of Wintner's theorem it is possible to construct a new complete Riemannian metric on $R^{n}$ with respect to which the righthand side of the ODE becomes uniformly bounded. The same situation takes place also for many other sufficient conditions of two-sided sort. Below in Theorem 2.2 we proof that if on a complete Riemannian manifold the right-hand side is uniformly bounded, the flow of ODE is complete. 
It turns out that the condition of boundedness of the right-hand side of ODE with respect to a complete Riemannian metric can be modified so that it becomes necessary and sufficient for completeness. This modification involves in particular transition to extended phase space.

Recall that in contemporary topology a map $f: X \rightarrow Y$ from a topological space $X$ to a topological space $Y$ is called proper, if the preimage of any relatively compact set from $Y$ is relatively compact in $X$. According to this terminology we give the following.

Definition 2.1. A function $f: X \rightarrow R$ on the topological space $X$ is called proper if the preimage of any relatively compact set from $R$ is relatively compact in $X$.

Recall that in a complete Riemannian manifold and so in an Euclidean space (in particular, in $R$ ) a set is relatively compact if and only if it is bounded.

We should mention that in $R^{n}$ a positive function $f$ is proper if and only if $f(x) \rightarrow+\infty$ as $\|x\| \rightarrow+\infty$. On a smooth manifold the Riemannian distance of any complete Riemannian metric is a proper function. Below we sometimes will not specify a Riemannian metric on $M$ and in this case the exact mathematical meaning of $x \rightarrow \infty$ for $x \in M$ is that $x$ leaves every compact set, that is, $f(x) \rightarrow \infty$ for any proper function $f$ on $M$.

Let $M$ be a finite-dimensional smooth manifold and $X(t, m)$ be a smooth (jointly in $t \in R$ and $m \in M$ ) vector field on $M$.

Denote by $m(s): M \rightarrow M, s \in R$ the flow of $X$. For any point $x \in M$ and time instant $t$ the orbit $m(s)(t, m)=m_{t, m}(s)$ of the flow is the solution of

$$
\dot{m}_{t, m}(s)=\left(s, m_{t, m}(s)\right)
$$

with the initial condition

$$
m_{t, m}(t)=m
$$

The orbits are also called the integral curves of $X$.

Consider the extended phase space $M^{+}=R \times M$ and the vector field $X_{(t, m)}^{+}=(1, X(t$, $m)$ ) on it.

Theorem 2.2 (see [6]). The flow of $X$ on $M$ is complete if and only if there exists a complete Riemannian metric on $\mathrm{M}^{+}$with respect to which the vector field $\mathrm{X}^{+}$is uniformly bounded.

Proof. It is evident that the completeness of flow for $X$ is equivalent to the completeness of flow for $X^{+}$.

Sufficiency. Let on $\mathrm{M}^{+}$there exist a complete Riemannian metric with respect to which the field $X^{+}$is uniformly bounded. Then any integral curve of $X^{+}$on any finite time interval has finite length and by completeness of the metric it is relatively compact, that is, the domain of solution is both closed and open in $R$. Hence it coincides with $R$.

Necessity. Let the vector field $X$ be complete. Then $X^{+}$is also complete. Since $X(t, m)$ is smooth by the hypothesis, $X^{+}$is also smooth. Following [12], let us construct a certain proper function $\psi$ on in the following way. Take on $M$ a countable locally finite covering 
$\mathscr{V}=\left\{V_{i}\right\}_{i \in N}$ where all $V_{i}$ are open and relatively compact. This can be done by virtue of the paracompactness and the local compactness of $M$. Determine $\psi_{i}: M \rightarrow R$ by the formula

$$
\psi_{i}(m)= \begin{cases}i & \text { if } m \in V_{i} \\ 0 & \text { if } m \notin V_{i}\end{cases}
$$

By $\left\{\phi_{i}\right\}_{i=1}^{\infty}$ denote the smooth partition of unity corresponding to this covering. Define the function $\psi$ on the entire $M$ as $\psi(m)=\sum_{i=1}^{\infty} \psi_{i}(m) \phi_{i}(m)$. It is clear that $\psi(m)$ is smooth and proper by the construction.

In every tangent space $T_{(t, m)}(\{t\} \times M)$ to the submanifold $\{t\} \times M$ of $M^{+}$introduce a scalar product that smoothly depends on $(t, m)$. For example, one can take an arbitrary Riemannian metric on $M$ and extend it by natural way. Now construct the Riemannian metric on $M^{+}$by regarding the vectors of the field $X^{+}$as being of unit length and orthogonal to the subspaces $T_{(m, t)}(M \times\{t\})$.

Consider the function $\Phi(t, m)=\psi\left(m_{(t, m)}^{+}(0)\right)$ on $M^{+}$, where $m_{(t, m)}^{+}(s)$ is the integral curve of $X^{+}$with initial condition $m_{(t, m)}^{+}(t)=m$ (the orbit of flow $m^{+}(s)$ corresponding to the vector field $X^{+}$on $M^{+}$). Since by the hypothesis the integral curves of $X^{+}$exist on $(-\infty, \infty)$, the function $\varphi: M^{+} \rightarrow R$, given by the formula $\varphi(t, m)=\Phi(t, m)+t$, is obviously well-posed smooth and proper. It is also obvious that $X^{+} \varphi=1\left(X^{+} \varphi\right.$ is the derivative of $\varphi$ in the direction of $X^{+}$).

Now pick an arbitrary smooth function $g: M^{+} \rightarrow R$ such that $g(t, m)>\max _{\|Y\|_{1}=1} \exp$ $(Y \varphi)^{2}, Y \in T_{(t, m)}(\{t\} \times M)$. Such a function can by constructed, for example, as follows. For a relatively compact neighborhood of any point $\left(t^{\prime}, m^{\prime}\right) \in M^{+}$there exists a constant that is greater than $\sup \max _{\|Y\|_{1}=1} \exp (Y \varphi)^{2}, Y \in T_{(t, m)}(\{t\} \times M)$ for all points $(t, m)$ from this neighborhood. Taking into account paracompactness of $M^{+}$and so, the existence of a smooth partition of unity (as above) from those constants we can glue the function $\phi$ defined on the whole of $M^{+}$.

At every point $(m, t) \in M^{+}$, define the inner product on $T_{(m, t)} M^{+}$by the formula

$$
\langle Y, Z\rangle_{2}=g^{2}(t, m)\left\langle p_{m} Y, p_{m} Z\right\rangle_{1}+p_{x} Y p_{x} Z,
$$

where $Y, Z \in T_{(m, t)} M^{+}$and $p_{m}, p_{X}$ are orthogonal (in the metric $\langle\cdot, \cdot\rangle_{1}$ ) projections of $T_{(m, t)} M^{+}$onto $T_{(t, m)}(\{t\} \times M)$ and $X^{+}$, respectively.

It is obvious that in the metric $\langle\cdot, \cdot\rangle_{2}$ the vector $X$ is still orthogonal to the subspace $T_{(t, m)}(\{t\} \times M)$ and $\|X\|_{2}=1$.

Lemma 2.3. $\langle\cdot, \cdot\rangle_{2}$ is a complete Riemannian metric on $\mathrm{M}^{+}$.

Proof of Lemma 2.3. By Hopf-Rinow theorem (see, e.g., [2]) it is sufficient to show that any geodesic of the metric $\langle\cdot, \cdot\rangle_{2}$ exists on $(-\infty, \infty)$. It is enough to deal with the geodesics whose norm of velocity vector is equal to 1 (all others can be obtained from them by linear change of argument). Let $c(s)$ be such a geodesic, that is, $\|\dot{c}(s)\|_{2}=1$ for all $s$. It is easy to see that $(d / d s) \varphi(c(s))=\dot{c}(s) \varphi=\left(p_{m} \dot{c}(s)\right) \varphi+\left(p_{x} \dot{c}(s)\right) \varphi$ (recall that here $\dot{c}(s) \varphi$ denotes the derivative of $\varphi$ in the direction of $\dot{c}(s)$; for $\left(p_{m} \dot{c}(s)\right) \varphi$ and $\left(p_{x} \dot{c}(s)\right) \varphi$ the meaning is analogous). Since $\|\dot{c}(s)\|_{2}=1$ and the vectors $p_{m} \dot{c}(s)$ and $p_{x} \dot{c}(s)$ are orthogonal to each 
other in the metric $\langle\cdot, \cdot\rangle_{2}$, we have $\left\|p_{m} \dot{c}(s)\right\|_{2} \leq 1,\left\|p_{x} \dot{c}(s)\right\|_{2} \leq 1$. Hence

$$
\left|\frac{d}{d s} \varphi(c(s))\right| \leq\left|\frac{p_{m} \dot{c}(s)}{\left\|p_{m} \dot{c}(s)\right\|_{2}} \varphi\right|+\left|\frac{p_{x} \dot{c}(s)}{\left\|p_{x} \dot{c}(s)\right\|_{2}} \varphi\right|=\left|\frac{1}{g(c(s))} \frac{p_{m} \dot{c}(s)}{\left\|p_{m} \dot{c}(s)\right\|_{1}} \varphi\right|+\left|X^{+} \varphi\right|<2
$$

by the constructions of functions $g$ and $\varphi$.

Thus, the values of $\varphi(c(s))$ are bounded on any finite interval $s \in(a, b)$ and the set of points $c(s)$ for $s \in(a, b)$ is relatively compact since $\varphi$ is proper. This proves the existence of geodesics on $(-\infty, \infty)$.

As it is mentioned above, $\left\|X^{+}\right\|_{2}=1$. The theorem follows.

Remark 2.4. Let us emphasize that for the case of an autonomous smooth vector field $X$ a complete metric on the manifold $M$, with respect to which $X$ is uniformly bounded, may not exist.

Indeed, consider in $R^{n}$ two differential equations $\dot{x}=\|x\|^{2} \cdot x$ and $\dot{x}=-\|x\|^{2} \cdot x$, where $\|x\|$ is the Euclidean norm of $x \in R^{n}$. It is well known that the field $-\|x\|^{2} \cdot x$ is complete while the field $\|x\|^{2} \cdot x$ is not complete: all its integral curves go to infinity within finite time interval. Nevertheless, those fields differ from each other only by the sign, that is, with respect to any Riemannian metric on $M$ their norms are equal to each other.

\section{Necessary and sufficient conditions of one-sided type for completeness of ODE flows}

As well as in Section 2 consider a smooth manifold $M$ with dimension $n<\infty$ and a smooth jointly in $t \in R, m \in M$ vector field $X=X(t, m)$ on $M$. The coordinate representation in a chart with respect to local coordinates $\left(q^{1}, \ldots, q^{n}\right)$ takes the form $X=$ $X^{1}\left(\partial / \partial q^{1}\right)+\cdots+X^{n}\left(\partial / \partial q^{n}\right)$. The vector field $X$ can be also considered as the first-order differential operator on $C^{1}$-functions on $M$. For a function $f$ the value of the above operator is given as $X f=X^{1}\left(\partial f / \partial q^{1}\right)+\cdots+X^{n}\left(\partial f / \partial q^{n}\right)$, the derivative of $f$ in the direction of vector field $X$. Let $\gamma(t)$ be an integral curve of $X$ such that $\gamma(0)=m$. It is well known that $X f$ is represented in terms of $\gamma(t)$ as follows: $X f(m)=\left.(d / d t) f(\gamma(t))\right|_{t=0}$. The latter presentation is valid also in infinite-dimensional case where the use of coordinates is not applicable.

Consider the extended phase space $M^{+}=R \times M$ with the natural projection $\pi^{+}: M^{+} \rightarrow$ $M, \pi^{+}(t, m)=m$. As well as in Section 2 introduce the vector field $X_{(t, m)}^{+}=(1, X(t, m))$ on $M^{+}$. It is clear that its coordinate representation is given in the form $X^{+}=\partial / \partial t+$ $X^{1}\left(\partial / \partial q^{1}\right)+\cdots+X^{n}\left(\partial / \partial q^{n}\right)$. Hence the corresponding differential operator on the space of $C^{1}$-smooth functions on $M^{+}$takes the form $\partial / \partial t+X$.

Theorem 3.1 (see [10]). A smooth vector field $X$ on a finite-dimensional manifold $M$ is complete if and only if there exists a smooth proper function $\varphi: M^{+} \rightarrow R$ such that the absolute value of derivative $\left|X^{+} \varphi\right|$ of $\varphi$ along $X^{+}$is uniformly bounded, that is, $\left|X^{+} \varphi\right|=$ $|(\partial / \partial t+X) \varphi| \leq C$ at any $(t, m) \in M^{+}$for a certain constant $C>0$ that does not depend on $(t, m)$. 
Proof

Sufficiency. Consider the flow $m^{+}(s): M^{+} \rightarrow M^{+}, s \in R$ with orbits $m^{+}(s)(t, m)=m_{(t, m)}^{+}(s)$ being the solutions of

$$
\dot{m}_{(t, m)}^{+}(s)=X^{+}\left(m_{(t, m)}^{+}(s)\right)
$$

with initial conditions

$$
m_{(t, m)}^{+}(t)=(t, m)
$$

Consider the derivative $X^{+} \varphi$ of $\varphi$ along $X^{+}$where $\varphi$ is from the hypothesis. At $(t, m) \in$ $M^{+}$we get the equality

$$
X^{+} \varphi(t, m)=\left.\frac{d}{d s} \varphi\left(m_{(t, m)}^{+}(s)\right)\right|_{s=t},
$$

(see above) and under the hypothesis of our theorem

$$
\left|\frac{d}{d s} \varphi\left(m_{(t, m)}^{+}(s)\right)\right|_{s=t} \mid \leq C
$$

Represent the values of $\varphi$ along the orbit $m_{(t, m)}^{+}(s)$ as follows:

$$
\varphi\left(m_{(t, x)}^{+}(s)\right)-\varphi(t, m)=\int_{0}^{s} \frac{d}{d \tau} \varphi\left(m_{(t, m)}^{+}(\tau)\right) d \tau
$$

From the last equality and from inequality (3.4) we evidently obtain that if $s$ belongs to a finite interval, the values $\varphi\left(m_{(t, x)}^{+}(s)\right)$ are bounded in $R$. Then since $\varphi$ is proper, this means that the set $m_{(t, m)}^{+}(s)$ is relatively compact in $M^{+}$.

Recall that by the classical solution existence theorem the domain of any solution of ODE is an open interval in $R$. In particular, for $s>0$ the solution of above Cauchy problem is well-posed for $s \in[t, \varepsilon)$. If $\varepsilon>0$ is finite, then the corresponding values of the solution belong to a relatively compact set in $M$ and so the solution is well-posed for $s \in[t, \varepsilon]$. The same arguments are valid also for $s<0$. Thus, the domain is both open and closed and so it coincides with the entire real line $(-\infty, \infty)$.

Taking into account the construction of vector field $X^{+}$, we can represent the integral curves $m_{(t, m)}^{+}(s)$ in the form $m_{(t, m)}^{+}(s)=\left(s, m_{t, m}(s)\right)$. Hence from global existence of integral curves of $X^{+}$we obviously obtain the global existence of integral curves of $X$. So, the vector field $X$ is complete.

Necessity. Let the vector field $X$ be complete. Thus, all orbits $m_{t, m}(s)$ of the flow $m(s)$ are well-posed on the entire real line.

Consider the function $\varphi: M^{+} \rightarrow R$ as in the proof of Theorem 2.2. As well as in the proof of Theorem 2.2 from completeness of $X^{+}$it follows that $\varphi$ is well-posed smooth 
and proper. Consider $X^{+} \varphi$. By the construction of the vector field $X^{+}$and of the function $\varphi$, we get

$$
X^{+} \varphi=X^{+}(\Phi(t, m))+X^{+} t=0+1=1
$$

Thus, we have proven the necessity part of our theorem for $C=1$. This completes the proof.

\section{A generalization to infinite-dimensional case}

Both Theorems 2.2 and 3.1 cannot be generalized to the infinite-dimensional case directly. For Theorem 2.2 the fatal difficulty is the absence of good enough infinite-dimensional analogy of Hopf-Rinow theorem. For Theorem 3.1 the main difficulty is the absence of continuous proper real-valued functions on infinite-dimensional manifolds. However it is possible to replace the set of functions, proper with respect to strong topology, by the one, proper with respect to a weaker topology so that an analogue of Theorem 3.1 takes place.

Let $M$ be a Banach manifold that admits partition of unity of class $C^{p}$ for a certain $p \geq 2$ (see [13]).

For the sake of convenience we consider charts on $M$ as triples $(U, V, \varphi)$, where $V$ is an open ball in the model space, $U$ is an open set in $M$, and $\varphi: V \rightarrow U$ is a homeomorphism.

Definition 4.1. A set $\Theta$ on $M$ is called relatively weakly compact if there exists a finite collection of charts $\left(U_{i}, V_{i}, \varphi_{i}\right)$ such that $\Theta \subset \bigcup_{i} U_{i}$ and for every $i$ the set $\varphi_{i}^{-1}\left(\Theta \cap U_{i}\right) \subset V_{i}$ is bounded with respect to the norm of model space that contains $V_{i}$.

Remark 4.2. If the model space of $M$ is a reflexive Banach space, then under some natural condition the relatively weakly compact set as in Definition 4.1 is relatively weakly compact with respect to the topology of weal convergence on $M$ (see [15]). If $M$ itself is a reflexive Banach space, then any relatively weakly compact set as in Definition 4.8 is weakly compact by standard definition of weak topology. These circumstances allow us to use the term "relatively weakly compact set" in the general case of Banach manifolds where (generally speaking) the weak topology is ill-posed.

Definition 4.3. A function $f: N \rightarrow R$ on a Banach space $N$ is called weakly proper if for any relatively compact set in $R$ its preimage is relatively weakly compact in $N$ as in Definition 4.1

Let $X=X(t, m)$ be a smooth jointly in $t \in R, m \in M$ vector field on $M$. Consider the extended phase space $M^{+}=R \times M$ and the vector field $X_{(t, m)}^{+}=(1, X(t, m))$ on it (cf. Sections 2 and 3 ).

Now we are in the position to prove the following generalization of Theorem 3.1.

Theorem 4.4 (see [11]). Let $M$ be a Banach manifold that admits partition of unity of class $C^{p}$ for a certain $p \geq 2$. A smooth vector field $X$ on $M$ is complete if and only if there exists a $C^{2}$-smooth weakly proper function $f: M^{+} \rightarrow R$ on $M^{+}$such that the absolute value of the derivative of $f$ in the direction of $X^{+}$is uniformly bounded, that is, $\left|X^{+} f\right| \leq C$ for a certain constant $C>0$ that does not depend on $(t, m)$. 
Proof

Sufficiency. Let there exist $f$ as in the hypothesis. Consider the flow $m^{+}(s): M^{+} \rightarrow M^{+}$, $s \in R$ of $X^{+}$. Its orbits $m^{+}(s)(t, m)=m_{t, m}^{+}(s)$ satisfy

$$
m_{(t, m)}^{++}(s)=X^{+}\left(m_{(t, m)}^{+}(s)\right)
$$

with initial conditions

$$
m_{(t, m)}^{+}(t)=(t, m)
$$

Show the existence of all orbits on $s \in(-\infty, \infty)$. Consider the derivative $X^{+} f$. At the point $(t, m)$ the equality

$$
X^{+} f(t, m)=\frac{d}{d s} f\left(m_{(t, m)}^{+}(s)\right)_{\mid s=t}
$$

holds and by the hypothesis

$$
\left|\frac{d}{d s} f\left(m_{(t, m)}^{+}(s)\right)_{\mid s=t}\right| \leq C
$$

Thus, on any finite interval $[t, \varepsilon)$ the values $f\left(m_{(t, m)}^{+}(s)\right)$ are bounded by the constant $C(\varepsilon-t)$. Then from Definitions 4.1 and 4.3 it follows that for $s \in(0, \varepsilon)$ there exists a finite number of charts $\left(U_{i}, V_{i}, \varphi_{i}\right)$ such that the set $m_{(t, m)}^{+}(s)$ belongs to the union of $U_{i}$ and the part of corresponding set in any $V_{i}$ is bounded. In particular, the part in the last $V_{i}$ is bounded and so there exists $\lim _{s \rightarrow \varepsilon}\left(m_{(t, m)}^{+}(s)\right)$, that is, $m_{(t, m)}^{+}(s)$ does exist on the closed interval $[t, \varepsilon]$. As well as in finite-dimensional situation this means that the domain of $m_{(t, m)}^{+}(s)$ is the entire $R$. Obviously, $m_{(t, m)}^{+}(s)=\left(s, m_{(t, m)}(s)\right)$. Hence from the completeness of $X^{+}$it follows that $X$ is also complete.

Necessity. Let $X$ be complete, that is, all orbits $m_{(t, m)}(s)$ exist on the entire line. Then all orbits of the flow $m^{+}(s)$ also exist on the entire line.

Construct an open covering of $M$ in the following way. For any $m \in M$ pick a chart $\left(U_{m}, V_{m}, \varphi_{m}\right)$ such that $m \in U_{m}$. Pick also an open neighborhood $W_{m} \subset U_{m}$ of $m$ such that $\varphi^{-1}\left(W_{m}\right) \subset V_{m}$ is bounded with respect to the norm of model space where $V_{m}$ is contained. Notice that by the construction $W_{m}$ is relatively weakly compact. Since $M$ is paracompact and satisfies the second axiom of countability, we can choose from $\left\{W_{m}\right\}$ a countable locally finite subcovering $\left\{W_{i}\right\}$ (see [13]).

Define the functions $\psi_{i}: M \rightarrow R$ by the formula

$$
\psi_{i}(m)= \begin{cases}i & m \in W_{i} \\ 0 & m \notin W_{i} .\end{cases}
$$

By $\theta_{i}(m), i=1, \ldots, \infty$, denote the $C^{p}$-smooth partition of unity, corresponding to $\left\{W_{i}\right\}$, 
that exists by the hypothesis. Define the function $\psi(m)$ on $M$ by the formula

$$
\psi(m)=\sum_{i=1}^{\infty} \theta_{i}(m) \psi_{i}(m) .
$$

By the construction $\psi(m)$ is $C^{p}$-smooth and weakly proper.

Now construct the $C^{P}$-smooth function $\Phi: M^{+} \rightarrow R$ by assigning to the point $(t, m) \in$ $M^{+}$the value $\Phi(t, m)=\psi\left(m_{t, m}(0)\right)$. Since $X^{+}$is complete, $\Phi(t, m)$ is well-posed.

By its construction the function $\Phi$ takes constant values along the orbits of $X^{+}$. Indeed, for $m_{(t, m)}^{+}(s)=\left(s, m_{(t, m)}(s)\right)$ the equality $m_{\left(s, m_{(t, m)}(s)\right)}(0)=m_{t, m}(0)$ holds. Consider the function $f: M^{+} \rightarrow R, f(t, m)=\Phi(t, m)+t$ that is $C^{p}$-smooth and weakly proper by the construction. Taking into account the construction of $X^{+}$and $f$, we get

$$
X^{+} f=X^{+} \Phi(t, m)+X^{+} t=0+1=1 .
$$

Thus, any $C \geq 1$ can be chosen as the constant from the assertion of theorem that we are looking for.

\section{Stochastic case}

The results of this section were announced in $[9,7]$.

Let $M$ be a finite-dimensional noncompact manifold. Consider a smooth stochastic dynamical system (SDS) on $M$ (see [3]) with the infinitesimal generator $\mathscr{A}(x)$. In local coordinates it is described in terms of a stochastic differential equation with $C^{\infty}$-smooth coefficients in Itô or in Stratonovich form. Since the coefficients are smooth, we can pass from Stratonovich to Itô equation and vice versa.

Consider the one-point compactification $M \bigcup\{\infty\}$ of $M$ where the system of open neighbourhoods of $\{\infty\}$ consists of complements to all compact sets of $M$. Denote by $\xi(s): M \rightarrow M \bigcup\{\infty\}$ the stochastic flow of SDS. For any point $x \in M$ and time $t$ the orbit $\xi_{t, x}(s)$ of this flow is the unique solution of the above-mentioned equation with initial conditions $\xi_{t, x}(t)=x$. As the coefficients of equation are smooth, this is a strong solution and a Markov diffusion process given on a certain random time interval. The point $\{\infty\}$ is the "cemetery" where the solution (defined on a random time interval) gets after explosion.

We refer the reader to [14] for more information on behavior of a diffusion process at infinity.

Recall that the generator $\mathscr{A}$ is a second-order differential operator without constant term (i.e., $\mathscr{A} 1=0$ where 1 denotes the constant function identically equal to 1 ). In local coordinates one can find the matrix of its pure second-order term that is symmetric and so semipositive definite.

For a stochastic flow the generator plays the same role as the derivative in the direction of vector field in the right-hand side of an ordinary differential equation. The main result on completeness for stochastic flows here is analogous to Theorem 3.1 where the derivative in the direction of vector field $X^{+}$is replaced with the corresponding generator. However, in the stochastic case there is an additional difficulty that for a flow with inverse 
time direction the generator does not coincide with the one for the flow itself. That is why we obtain a necessary and sufficient condition for completeness only for flows with additional assumption: the flow must be continuous at infinity (see the exact definition below).

Everywhere in this section we suppose $\mathscr{A}(x)$ to be autonomous and strictly elliptic (i.e., in a local coordinate system its pure second-order term is described by a nondegenerate, i.e., positive definite matrix). This assumption allows us to apply the machinery from [1]. Notice that using this machinery we can reduce the condition that the SDS is $C^{\infty}$-smooth to the assumption that it is Hölder continuous.

Below we denote the probability space, where the flow is defined, by $(\Omega, \mathscr{F}, \mathrm{P})$ and suppose that it is complete. We also deal with separable realizations of all processes.

Let $T>0$ be a real number.

Definition 5.1. The flow $\xi(s)$ is complete on $[0, T]$ if $\xi_{t, x}(s)$ is a.s. in $M$ for any couple $(t, x)$ (with $0 \leq t \leq T$ ) and for all $s \in[t, T]$.

Definition 5.2. The flow $\xi(s)$ is complete if it is complete on any interval $[0, T] \subset R$.

We start with a certain sufficient condition for completeness of a stochastic flow analogous to conditions for completeness of ODE flows with one-sided estimates. It is a simple version of rather general sufficient condition from [3, Theorem IX. 6A].

Theorem 5.3. Let there exist a smooth proper function $\varphi$ on $M$ such that $\mathscr{A}(t, m) \varphi<C$ for some $C>0$ at all $t \in[0,+\infty)$ and $m \in M$. Then the flow $\xi(t, s)$ is complete.

Proof. Consider the collection of sets $W_{n}=\varphi^{-1}([0, n))$ with the positive integers $1,2, \ldots$, $n, \ldots$ Since $\varphi$ is proper, those sets are relatively compact and $\bigcup_{n} W_{n}=M$. Besides, by the construction $W_{i} \subset W_{i+1} i=1,2, \ldots, n, \ldots$

Specify arbitrary $t \in[0,+\infty)$ and $m \in M$ and consider the orbit $\xi_{t, m}(s)$. Denote by $\tau_{n}$ the first entrance time of $\xi_{t, m}(s)$ in the boundary of $W_{n}$. Express $\varphi\left(\xi_{t, m}\left(s \wedge \tau_{n}\right)\right)$ by Itô formula. Since $W_{n}$ is relatively compact, Itô integral on the interval $\left[t, s \wedge \tau_{n}\right.$ ) is a martingale and so its expectation is equal to 0 . Then

$$
E \varphi\left(\xi_{t, m}\left(s \wedge \tau_{n}\right)\right)=\varphi(m)+\int_{t}^{s \wedge \tau_{n}}(\mathscr{A} \varphi)\left(\theta, \xi_{t, m}(\theta)\right) d \theta<\varphi(m)+C s
$$

since $\mathscr{A}(t, m) \varphi<C$ and $s \geq s \wedge \tau_{n}$.

Consider the set $\Omega_{s}^{n}=\left\{\omega \in \Omega \mid s<\tau_{n}\right\}$. Obviously,

$$
n\left(1-\mathrm{P}\left(\Omega_{s}^{n}\right)\right)<E \varphi\left(\xi_{t, m}\left(s \wedge \tau_{n}\right)\right)
$$

since for $\omega \notin \Omega_{s}^{n}$ we get $\xi_{t, m}\left(s \wedge \tau_{n}, \omega\right)=\xi_{t, m}\left(\tau_{n}, \omega\right)$, that is, $\varphi\left(\xi_{t, m}\left(s \wedge \tau_{n}, \omega\right)\right)=n$. Thus,

$$
1-\mathrm{P}\left(\Omega_{s}^{n}\right)<\frac{\varphi(m)+C s}{n} .
$$

Hence $\lim _{n \rightarrow \infty}\left(1-\mathrm{P}\left(\Omega_{s}^{n}\right)\right)=0$. However by the construction $\lim _{n \rightarrow \infty} \Omega_{s}^{n}=\bigcup_{i=1}^{n} \Omega_{s}^{i}=\Omega$, that is, for any specified $s \geq t$ the value $\xi_{t, m}(s)$ exists with probability 1 . 
Let $\gamma(s)$ be a (not necessarily complete) stochastic flow.

Definition 5.4. We say that the flow $\gamma(s)$ is continuous at infinity if for any $0 \leq t \leq T$ and any compact $K \subset M$ the equality

$$
\lim _{x \rightarrow+\infty} \mathrm{P}\left(\gamma_{t, x}(T) \in K\right)=0
$$

holds.

One can easily see that continuity at infinity according to Definition 5.4 means that for any specified $t \in[0,+\infty)$ and for all $s \in[t,+\infty)$ the correspondence $(x, s) \mapsto \gamma_{t, x}(s)$ is continuous in probability at the point $(s,\{\infty\}) \in[t, \infty] \times(M \bigcup\{\infty\})$, see [16, 17] for details.

Our next task is to construct a special proper function associated to a complete stochastic flow $\xi(s)$.

Consider an expanding sequence of compact sets $M_{i}$ such that $M_{i} \subset M_{i+1}$ for all $i$ and $\bigcup_{i} M_{i}=M$. By $T_{i}$ we denote an increasing sequence of real numbers tending to $+\infty$.

For $(t, x) \in\left[0, T_{i}\right] \times M_{i}$, the distribution function $\mu_{t, x, s}$ of random elements $\xi_{t, x}(s), s \in$ $\left[t, T_{i}\right]$, on $M$ forms a weakly compact set of measures. Indeed, take an arbitrary sequence random element $\xi_{t_{k}, x_{k}}\left(s_{k}\right)$ and the corresponding measures $\mu_{t_{k}, x_{k}, s_{k}}$. Since $\left[0, T_{i}\right] \times M_{i} \times$ $\left[0, T_{i}\right]$ is compact, it is possible to select a subsequence $t_{k_{q}}, x_{k_{q}}, s_{k_{q}}$ of the sequence $t_{k}, x_{k}$, $s_{k}$, converging to a certain $t_{0}, x_{0}, s_{0}$. It is a well-known fact that the function $\operatorname{Ef}\left(\xi_{t, x}(s)\right)$ is continuous jointly in $t, x, s$ for any bounded continuous function $f: M \rightarrow R$. Then we obtain that

$$
E\left(f\left(\xi_{t_{k_{q}}, x_{k_{q}}}\left(s_{k_{q}}\right)\right)\right) \longrightarrow E\left(f\left(\xi_{t_{0}, x_{0}}\left(s_{0}\right)\right)\right)
$$

that is, from any sequence of measures mentioned above it is possible to select a weakly converging subsequence.

Take a monotonically decreasing sequence of positive numbers $\varepsilon_{i} \rightarrow 0$ such that the series $\sum_{i=1}^{\infty} \sqrt{\varepsilon_{i}}$ converges. From Prokhorov's theorem it follows that for the measures corresponding to $\xi_{t, x}(s), s \in\left[t, T_{i}\right],(t, x) \in\left[0, T_{i}\right] \times M_{i}$ mentioned above, there exists a compact $\Xi_{i} \subset M$ such that for all $\mu_{t, x, s}$ the inequality $\mu_{t, x, s}\left(M \backslash \Xi_{i}\right)<\varepsilon_{i}$ holds. Construct an expanding system of compacts $\Theta_{i} \supset \bigcup_{k=0}^{i} \Xi_{k}$ for any $i$, being closures of open domains in $M$ with smooth boundary and such that $\Theta_{i} \subset \Theta_{i+1}$ for any $i$ and $\bigcup_{i} \Theta_{i}=M$. By the construction for $s \in\left[0, T_{i}\right],(t, x) \in\left[0, T_{i}\right] \times M_{i}$ the relation $\mu_{t, x, s}\left(M \backslash \Theta_{i}\right)<\varepsilon_{i}$ holds. In particular, $\mu_{t, x, s}\left(\Theta_{i+1} \backslash \Theta_{i}\right)<\varepsilon_{i}$.

Choose neighborhoods $U_{i} \subset \tilde{U}_{i}$ of the set $\Theta_{i}$, that completely belong to $\Theta_{i+1}$, and consider a smooth function $\psi_{i}$ that equals 0 on $U_{i}$, equals 1 on $\Theta_{i+1} \backslash \tilde{U}_{i}$, and takes values between 0 and 1 on $\tilde{U}_{i} \backslash U_{i}$. Construct the function $\theta$ on $M$ setting its value on $\Theta_{i+1} \backslash \Theta_{i}$ equal to $\psi_{i}\left(1 / \sqrt{\varepsilon_{i}}\right)+\left(1-\psi_{i}\right)\left(1 / \sqrt{\varepsilon_{i-1}}\right)$. Notice that on $\Theta_{i+1} \backslash \Theta_{i}$ the values of $\theta$ are not greater than $1 / \sqrt{\varepsilon_{i}}$.

Immediately from the construction we obtain the following.

Lemma 5.5. For a complete flow $\xi(s)$ the function $\theta$, constructed above, is smooth positive and proper. 
Theorem 5.6. If the flow $\xi(t)$ is complete, for any $(t, x)$ and any $T>t$, the inequality $E \theta\left(\xi_{t, x}(s)\right)<\infty$ holds for each $s \in[t, T]$.

Proof. Take $i$ such that $[0, T] \subset\left[0, T_{i}\right], t \in\left[0, T_{i}\right]$, and $x \in M_{i}$. Then $\mu_{t, x, s}\left(M \backslash \Theta_{i}\right)<\varepsilon_{i}$ or $\mu_{t, x, s}\left(\Theta_{i}\right)>\left(1-\varepsilon_{i}\right)$. By the construction the values of continuous function $\theta$ on compact $\Theta_{i}$ are bounded by constant $1 / \sqrt{\varepsilon_{i-1}}$. Then also by the construction

$$
E \theta\left(\xi_{t, x}(s)\right) \leq \frac{1}{\sqrt{\varepsilon_{i-1}}}+\sum_{k=i}^{\infty} \varepsilon_{k} \frac{1}{\sqrt{\varepsilon_{k}}}=\frac{1}{\sqrt{\varepsilon_{i-1}}}+\sum_{k=i}^{\infty} \sqrt{\varepsilon_{k}}<C<+\infty
$$

for some positive constant $C$ since by definition the series $\sum_{k=i+1}^{\infty} \sqrt{\varepsilon_{k}}$ converges.

Corollary 5.7. The function $E \theta\left(\xi_{t, x}(s)\right)$ is integrable in $s \in[t, T]$.

Proof. From the construction in Theorem 5.6 it follows that for given $t, x$ estimate (5.6) is valid with the same $C$ for all $s \in[t, T]$.

Specify any $T>0$ and consider the direct product $M^{T}=[0, T] \times M$. Denote by $\pi^{T}$ : $M^{T} \rightarrow M$ the natural projection: $\pi^{T}(t, x)=x$.

Theorem 5.8. The function $u(t, x)=E \theta\left(\xi_{t, x}(T)\right)$ on $M^{T}$ is $C^{1}$-smooth in $t \in[0, T], C^{2}$ smooth in $x \in M$ and satisfies

$$
\left(\frac{\partial}{\partial t}+\mathscr{A}\right) u=0
$$

where $\mathscr{A}$ is the infinitesimal generator of the flow.

Proof. Since $M$ is locally compact and paracompact, we can choose a countable locally finite open covering $\left\{V_{i}\right\}_{i=1}^{\infty}$ of $M$ such that all $V_{i}$ have compact closures. Consider a partition of unity $\left\{\varphi_{i}\right\}_{i=1}^{\infty}$ adapted to this covering. Then at any point $x \in M$ the equality $\theta(x)=\sum_{i=1}^{\infty} \varphi_{i}(x) \theta(x)$ holds.

Introduce the function $v_{i}(x)=\varphi_{i}(x) \theta(x)$ as well as the functions $u_{i}(t, x)=E v_{i}\left(\xi_{t, x}(T)\right)$ and $\theta_{k}(t, x)=\sum_{i=0}^{k} u_{i}(t, x)$. Notice that all $v_{i}(x)$ are smooth and bounded. Then any $v_{i}(t, x)$ satisfies the conditions of [ 5 , Theorem 4 , Chapter VIII] and so any $u_{i}(t, x)$ is $C^{1}$-smooth in $t, C^{2}$-smooth in $x$ and satisfies the relation

$$
\frac{\partial}{\partial t} u_{i}+\mathscr{A} u_{i}=0
$$

Hence all functions $\theta_{k}(t, x)$, being finite sums of functions $u_{i}(t, x)$, are also $C^{1}$-smooth in $t, C^{2}$-smooth in $x$ and satisfy

$$
\frac{\partial}{\partial t} \theta_{k}+\mathscr{A} \theta_{k}=0
$$

In addition it is evident that $\theta(t, x)$ is the limit of $\theta_{k}(t, x)$ at $k \rightarrow \infty$ and the functions $\theta_{k}(t, x)$ form an increasing locally bounded sequence. Then, since $\mathscr{A}$ is autonomous and strictly elliptic, the assertion of theorem follows from [1, Lemma 1.8]. 
Theorem 5.9. If a complete flow $\xi(s)$ is continuous at infinity, the function $u(t, x)=$ $E \theta\left(\xi_{t, x}(T)\right)$ on $M^{T}$ is proper.

Proof. Let $\xi(s)$ be continuous at infinity. To prove the properness of $u(t, x)$ it is sufficient to show that $u(t, x) \rightarrow \infty$ as $\theta(x) \rightarrow \infty$, that is, that for any $C>0$ there exists $\Xi>0$ such that $\theta(x)>\Xi$ yields $u(t, x)>C$ for any $t \in[0, T]$. Since $\theta$ is proper, $K=\theta^{-1}([0,2 C])$ is compact. From formula (5.4) of the definition of continuity at infinity it follows that for any $t \in[0, T]$ there exists $\Xi$ such that $\mathrm{P}\left(\xi_{t, x}(T) \notin K\right)>1 / 2$ for $\theta(x)>\Xi$. Then $u(t, x)=$ $E \theta\left(\xi_{t, x}(T)\right)>2 C \cdot 1 / 2=C$. Since $t$ is from compact set $[0, T]$ and $u(t, x)$ is continuous in $t$, this completes the proof.

On the manifold $M^{T}$ consider the flow $\eta(s)=(s, \xi(s))$. Obviously, for $(t, x) \in M^{T}$ the trajectory of $\eta_{(t, x)}(s)$ satisfies the relation $\pi^{T}\left(\eta_{(t, x)}(s)\right)=\xi_{t, x}(s)$. It is clear that $\eta(s)$ is the flow generated by SDS with infinitesimal generator $\mathscr{A}^{T}$ determined by the formula

$$
\mathscr{A}_{(t, x)}^{T}=\mathscr{A}(t, x)+\frac{\partial}{\partial t}
$$

Notice that $\mathscr{A}^{T}$ is a direct analogue of differentiation in the direction of $X^{+}$in Theorem 3.1 .

Theorem 5.10. A flow $\xi(s)$ on $M$, continuous at infinity, is complete on $[0, T]$ if and only if there exists a positive proper function $u^{T}: M^{T} \rightarrow R$ on $M^{T}$ that is $C^{1}$-smooth in $t \in[0, T]$, $C^{2}$-smooth in $x \in M$ and such that $\mathscr{A}^{T} u^{T}<C$ for a certain constant $C>0$ at all points $(t, x) \in M^{T}$.

Proof. Let there exist a smooth proper positive function $u^{T}(t, x)$ on $M^{T}$ such that $\mathscr{A}^{T} u^{T}$ $<C$ at all points of $M^{T}$. Then from Theorem 5.3 it follows that $\eta(s)$ is complete. Thus, $\xi(s)$ is also complete.

Let $\xi(s)$ be complete. Consider the function $\theta(x)$ on $M$ introduced above and the function $u^{T}(t, x)=E \theta\left(\xi_{t, x}(T)\right)$ on $M^{T}$. Since $\xi(s)$ is continuous at infinity, $u^{T}(t, x)$ is proper by Theorem 5.9. By Theorem 5.8 it is also $C^{1}$ in $t, C^{2}$ in $x$ and satisfies the relation $((\partial / \partial t)+\mathscr{A}) u^{T}=\mathscr{A}^{T} u^{T}=0$.

Corollary 5.11. A flow $\xi(s)$ on $M$, continuous at infinity, is complete if and only if for any $T>0$ there exists a positive proper function $u^{T}: M^{T} \rightarrow R$ on $M^{T}$ that is $C^{1}$-smooth in $t \in[0, T], C^{2}$-smooth in $x \in M$ and such that $\left|\mathscr{A}^{T} u(t, x)\right|<C$ for a certain constant $C>0$ at all points $(t, x) \in M^{T}$.

\section{Parabolic equations}

Here by using stochastic approach to parabolic equations and the results of Section 5 we get a necessary and sufficient condition for existence of global Feller semigroup for some class of such equation (in particular, this class includes equations with the so-called $C_{0}$ property). We suppose that the second-order operator in the right-hand side of parabolic equation is autonomous and strictly elliptic. Under this assumption, on the one hand, the stochastic approach is applicable and on the other hand, the conditions of Section 5 are fulfilled for the corresponding stochastic flow. 
Let $M$ be a finite-dimensional (generally speaking) noncompact manifold. Consider on $M$ a parabolic equation

$$
\frac{\partial}{\partial t} u=A u
$$

with initial conditions

$$
u(0, x)=u_{0}(x)
$$

where $\mathscr{A}$ is an autonomous strictly elliptic operator with $C^{\infty}$ coefficients without constant term (i.e., satisfying the property $\mathscr{A} 1=0), u_{0}$ and $u$ are smooth enough real-valued bounded functions.

In local coordinates on $M$, the operator $\mathscr{A}$ is represented in the form

$$
\sum_{i=1}^{n} a^{i} \frac{\partial}{\partial q^{i}}+\sum_{i=1}^{n} b^{i}\left(\sigma^{k l}\right) \frac{\partial}{\partial q^{i}}+\frac{1}{2} \sum_{i, j=1}^{n} \sigma^{i j} \frac{\partial^{2}}{\partial q^{i} \partial q^{j}} .
$$

Here $b_{x}(\sigma)=\sum_{i=1}^{n} b^{i}\left(\sigma^{k l}\right) \partial / \partial q^{i}$ is the so-called compensating term, depending on $\left(\sigma^{k l}\right)$, that guarantees covariant transformation of the formula under changes of coordinates.

It is a well-known fact that under the above conditions on $\mathscr{A}$ the stochastic approach to investigation of parabolic equations is applicable in the following way. One can easily see that the matrix $\left(\sigma^{i j}(x)\right)$ is a coordinate expression of a smooth symmetric $(2,0)$-tensor field on $M$. Since $\mathscr{A}$ is strictly elliptic, this matrix is not degenerate and taking at any $x \in M$ the inverse matrix $\left(\sigma_{i j}(x)\right)$ one gets a smooth $(0,2)$-tensor field. Denote the latter field by $\sigma_{x}$. Thus, $\sigma_{x}$ for any $x \in M$ is a symmetric bilinear form on the tangent space $T_{x} M$. Since $\mathscr{A}$ is strictly elliptic, this form at any $x \in M$ is positive definite and so the field $\sigma_{x}$ can be considered as a Riemannian metric tensor on $M$. By Nash's theorem we can embed $M$ with this metric isometrically into a certain Euclidean space $R^{k}$ where $k$ is large enough. Then the field of orthogonal projections $A_{x}: R^{k} \rightarrow T_{x} M$ is smooth and gives us the presentation of $\sigma_{x}$ in the form

$$
\sigma_{x}=A_{x}^{*} A_{x}
$$

where $A_{x}^{*}$ is the conjugate operator.

The above construction yields the existence of a smooth stochastic dynamical system (SDS) on $M$ (see [3]) whose infinitesimal generator is $\mathscr{A}$ and it is of the same type as in Section 5. In local coordinates it is described in terms of a stochastic differential equation with $C^{\infty}$-smooth coefficients in Itô or in Stratonovich form with diffusion term $A_{x}^{*}$. In Itô form its drift is $a+b$. Since the coefficients are smooth, we can pass from Itô form to Stratonovich one and vice versa.

Denote by $\xi(s)$ the flow of above-mentioned SDS and by $\xi_{t, x}(s)$ its orbits (see Section $5)$. If $\xi(s)$ is complete, on the space of bounded measurable functions on $M$, there exists an operator semigroup $S(t, s)$ given for a function $f(x)$ by the formula

$$
[S(t, s) f](x)=E f\left(\xi_{t, x}(s)\right)
$$


where $E$ is the mathematical expectation. This is a Feller semigroup, that is, for any $t \geq 0$, $s \geq t$ the operators $S(t, s)$ are contracting and transform any continuous positive bounded function into one with the same properties. It is also well known that for continuous and bounded function $u_{0}(x)$ the continuous and bounded function

$$
u(s, x)=\left[S(0, s) u_{0}\right](x)=E u_{0}\left(\xi_{0, x}(s)\right)
$$

is a generalized solution of (6.1)-(6.2). If $u(s, x)$ is smooth enough, it is a classical solution. By analytical methods it is shown that this solution is unique in the class of bounded measurable functions. See details, for example, in [4].

Thus, completeness of the stochastic flow $\xi(s)$ (i.e., global-in-time existence of solutions of the above-mentioned stochastic differential equation) is equivalent to global-intime existence of solutions of (6.1)-(6.2).

As a corollary to Theorem 5.10 and Corollary 5.11 we obtain the following theorem.

Theorem 6.1. If the flow $\xi(s)$ is continuous at infinity, the solutions of (6.1)-(6.2) exist globally in time if and only if for any $T>0$ there exists a positive proper function $v^{T}: M^{T} \rightarrow R$ that is $C^{1}$-smooth in $t \in[0, T], C^{2}$-smooth in $x \in M$ and such that $\mathscr{A}^{T} v^{T}(t, x)<C$ for a certain constant $C>0$ at all points $(t, x) \in M^{T}$.

Of course it is important to have conditions for global-in-time existence of solutions of $(6.1)-(6.2)$ without referring to the properties of corresponding flow $\xi(s)$. For this purpose we select a smaller class of equations according to the following.

Definition 6.2 (see [1]). The flow $\xi(s)$ and the corresponding semigroup $S$ are called to have $C_{0}$ property if for any compact $K \subset M$ the relation

$$
\lim _{x \rightarrow+\infty} \mathrm{P}\left(T_{K}\left(\xi_{t, x}\right)<T\right)=0
$$

holds where $T_{K}\left(\xi_{t, x}\right)$ is the first entrance time of $\xi_{t, x}$ in $K$.

It is well known that $C_{0}$ property is equivalent to the fact that the operators from semigroup $S$ leave invariant the space $C_{0}(M)$ of continuous functions, tending to zero at infinity (see, e.g., $[14,16,17]$ for details). Some conditions, under which $C_{0}$ property is satisfied, are found in [1].

Proposition 6.3. Any flow with $C_{0}$ property is continuous at infinity.

Proposition 6.3 follows from the obvious fact that $\mathrm{P}\left(T_{K}\left(\gamma_{t, x}\right)<T\right) \geq \mathrm{P}\left(\gamma_{t, x}(T) \in K\right)$. We refer the reader to $[14,16,17]$ for some details on behavior of a stochastic flow at infinity and on relations between $C_{0}$ property and continuity at infinity.

From Proposition 6.3, Theorem 5.10, and Corollary 5.11 we get the following.

THeorem 6.4. If operators (6.5) are $C_{0}$, the solutions of (6.1)-(6.2) exist globally in time if and only if for any $T>0$ there exists a positive proper function $v^{T}: M^{T} \rightarrow R$ that is $C^{1}$ smooth in $t \in[0, T], C^{2}$-smooth in $x \in M$ and such that $\mathscr{A}^{T} v^{T}(t, x)<C$ for a certain constant $C>0$ at all points $(t, x) \in M^{T}$. 


\section{Acknowledgments}

The research is partially supported by Grants 03-01-00112 and 04-01-00081 from RFBR. The author is indebted to K.D. Elworthy for very much useful discussions of the material of Section 5.

\section{References}

[1] R. Azencott, Behavior of diffusion semi-groups at infinity, Bulletin de la Société Mathématique de France 102 (1974), 193-240.

[2] R. L. Bishop and R. J. Crittenden, Geometry of Manifolds, Pure and Applied Mathematics, vol. 15, Academic Press, New York, 1964.

[3] K. D. Elworthy, Stochastic Differential Equations on Manifolds, London Mathematical Society Lecture Note Series, vol. 70, Cambridge University Press, Cambridge, 1982.

[4] M. I. Freidlin and A. D. Wentzell, Random Perturbations of Dynamical Systems, Fundamental Principles of Mathematical Sciences, vol. 260, Springer, New York, 1984.

[5] I. I. Gikhman and A. V. Skorohod, Introduction to the Theory of Random Processes, Nauka, Moscow, 1977.

[6] Yu. E. Gliklikh, On conditions of non-local prolongability of integral curves of vector fields, Differential equations 12 (1977), no. 4, 743-744 (Russian).

[7] _ A necessary and sufficient condition of completeness for a certain class of stochastic flows on manifolds, Trudy seminara po vektornomu i tenzornomu analizu, vol. 26, Moscow State University, Moscow, 2005, pp. 130-138.

[8] Global and Stochastic Analysis in Problems of Mathematical Physics, URSS (KomKniga), Moscow, 2005.

[9] _ A necessary and sufficient condition for completeness of stochastic flows continuous at infinity, Warwick preprint, August 2004, no. 8, pp. 1-7.

[10] Yu. E. Gliklikh and L. A. Morozova, Conditions for global existence of solutions of ordinary differential, stochastic differential, and parabolic equations, International Journal of Mathematics and Mathematical Sciences 2004 (2004), no. 17-20, 901-912.

[11] Yu. E. Gliklikh and A. V. Sinelnikov, On a certain necessary and sufficient condition for completeness of a vector field on a Banach manifold, Trudy matematicheskogo fakul'teta Voronezhskogo gosudarstvennogo universiteta, no. 9, 2005, pp. 46-50.

[12] C. Godbillon, Géométrie différentielle et mécanique analytique, Hermann, Paris, 1969.

[13] S. Lang, Differential Manifolds, Springer, New York, 1985.

[14] X.-M. Li, Properties at infinity of diffusion semigroups and stochastic flows via weak uniform covers, Potential Analysis 3 (1994), no. 4, 339-357.

[15] J.-P. Penot, Weak topology on functional manifolds, Global Analysis and Its Applications (Lectures, Internat. Sem. Course, Internat. Centre Theoret. Phys., Trieste, 1972), vol. 3, International Atomic Energy Agency, Vienna, 1974, pp. 75-84.

[16] L. Schwartz, Processus de Markov et désintégrations régulières, Université de Grenoble. Annales de l'Institut Fourier 27 (1977), no. 3, xi, 211-277.

[17] _ Le semi-groupe d'une diffusion en liaison avec les trajectoires, Séminaire de Probabilités, XXIII, Lecture Notes in Math., vol. 1372, Springer, Berlin, 1989, pp. 326-342.

Yuri E. Gliklikh: Faculty of Mathematics, Voronezh State University, Universitetskaya pl. 1, 394006 Voronezh, Russia

E-mail address: yeg@math.vsu.ru 


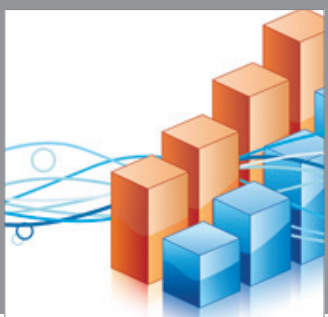

Advances in

Operations Research

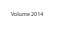

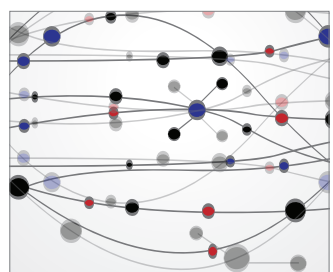

\section{The Scientific} World Journal
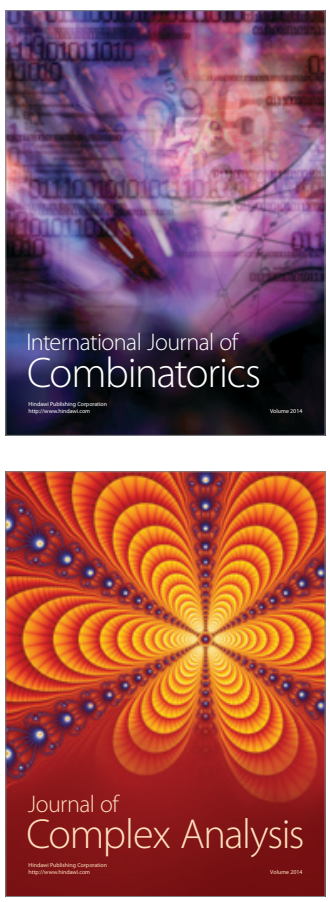

International Journal of

Mathematics and

Mathematical

Sciences
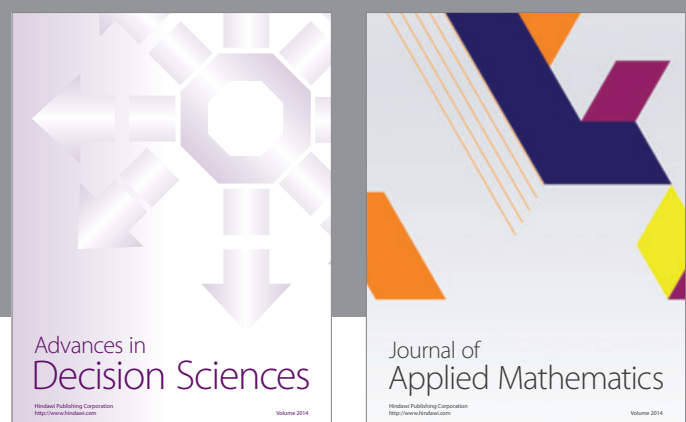

Journal of

Applied Mathematics
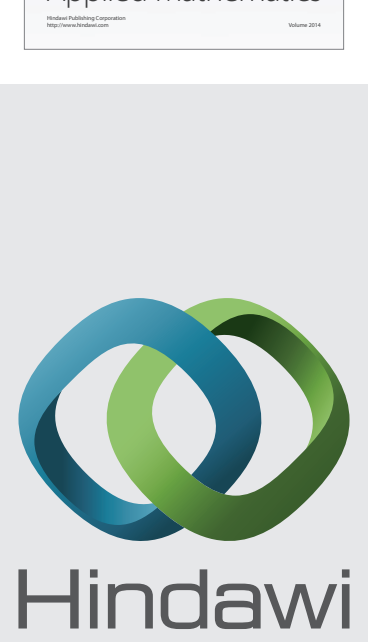

Submit your manuscripts at http://www.hindawi.com
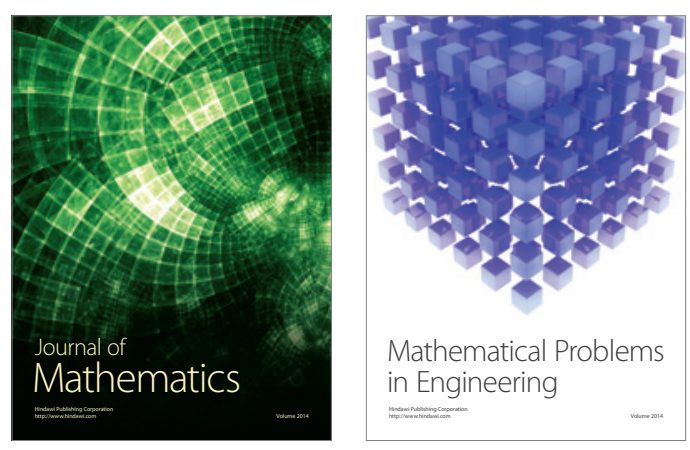

Mathematical Problems in Engineering
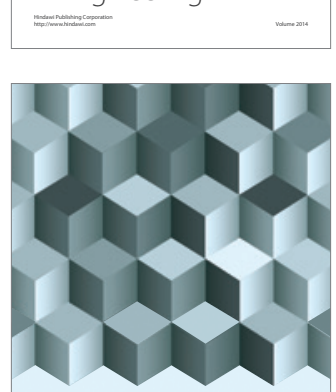

Journal of

Function Spaces
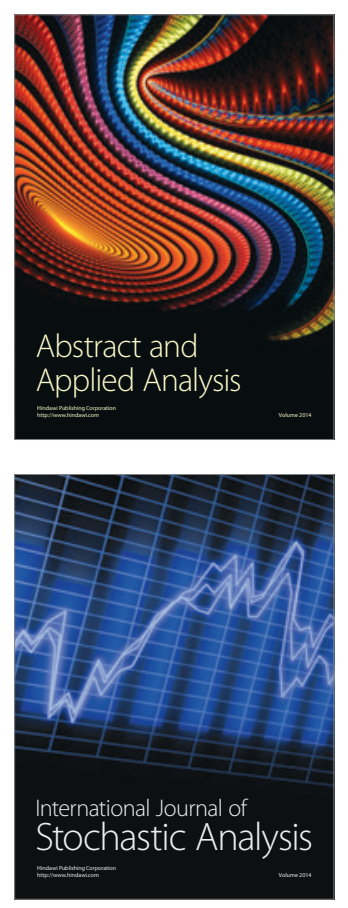

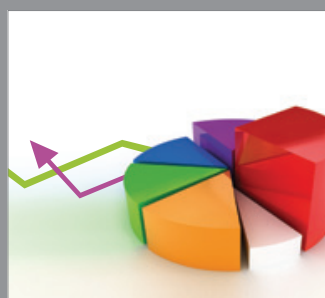

ournal of

Probability and Statistics

Promensencen
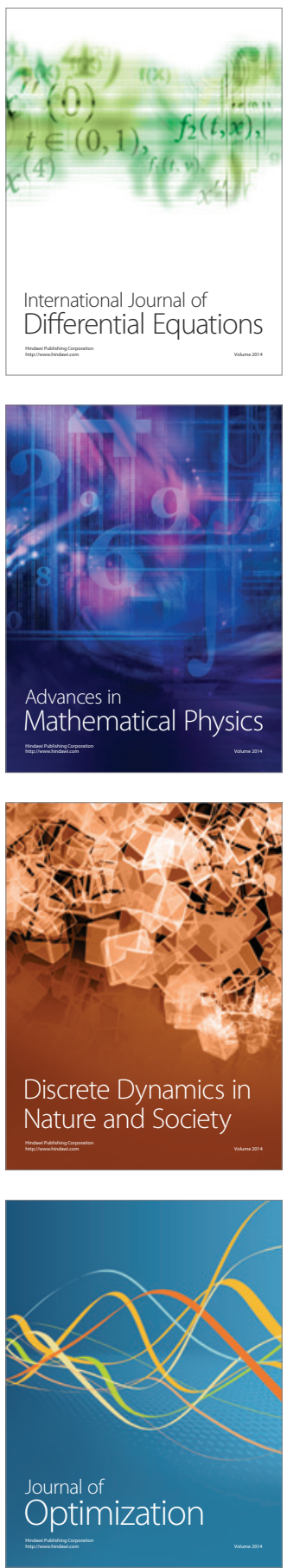